PROCEEDINGS OF THE

AMERICAN MATHEMATICAL SOCIETY

Volume 127, Number 2, February 1999, Pages 491-497

S 0002-9939(99)04540-2

\title{
ON A THEOREM OF E. HELLY
}

\author{
SAKAÉ FUCHINO AND SZYMON PLEWIK
}

(Communicated by Andreas R. Blass)

\begin{abstract}
E. Helly's theorem asserts that any bounded sequence of monotone real functions contains a pointwise convergent subsequence. We reprove this theorem in a generalized version in terms of monotone functions on linearly ordered sets. We show that the cardinal number responsible for this generalization is exactly the splitting number. We also show that a positive answer to a problem of S. Saks is obtained under the assumption of the splitting number being strictly greater than the first uncountable cardinal.
\end{abstract}

\section{INTRODUCTION}

E. Helly's theorem ([3]) asserts that any bounded sequence of monotone real functions contains a pointwise convergent subsequence. In the present paper, we prove the following generalization of the theorem: for linearly ordered sets $X$ and $Y$, if $Y$ is sequentially compact with density less than the splitting number $\mathbf{s}$, then any sequence of monotone functions from $X$ to $Y$ contains a pointwise convergent subsequence (Theorem 7 ). We also show that this theorem characterizes the splitting number (Theorem 9).

We begin with reviewing some definitions and elementary facts needed for our results.

\section{Preliminaries: Linearly ordered Sets}

A linearly ordered set $X$ is said to be dense linear order, if, for any $x, y \in X$, $x<y$ implies that there exists $z \in X$ such that $x<z<y$. A subset $D$ of a linearly ordered set $X$ is said to be dense in $X$, if, for any $x, y \in X, x<y$ implies that there exists $z \in D$ such that $x \leq z \leq y$. The density of $X$ is defined by

$$
d(X)=\min \{|D|: D \subseteq X \text { and } D \text { is dense in } X\},
$$

where $|D|$ denotes the cardinality of the set $D$.

Let $X$ and $Y$ be linearly ordered sets. A function $f: X \rightarrow Y$ is said to be increasing, if, for any $x, y \in X, x<y$ implies $f(x) \leq f(y)$; decreasing, if $x<y$ implies $f(y) \leq f(x)$. A function is monotone if it is either increasing or decreasing. A sequence $\left(x_{n}\right)_{n \in \mathbb{N}}$ of elements of $X$ is called increasing, decreasing or monotone respectively, if it is increasing, decreasing or monotone respectively as a function from the set of all natural numbers $\mathbb{N}$ into $X$.

Received by the editors August 8, 1996 and, in revised form, May 26, 1997.

1991 Mathematics Subject Classification. Primary 26A03, 06A05, 03E10, 03E35.

Key words and phrases. Helly's theorem, splitting number, Saks' problem.

(c)1999 American Mathematical Society 
For a linearly ordered set $X$, the notion of convergence can be introduced in a canonical way: an increasing sequence $\left(x_{n}\right)_{n \in \mathbb{N}}$ in $X$ converges to a point $x$, if $x$ is the supremum of the set of all elements of this sequence; a decreasing sequence converges to $x$, if $x$ is the infimum of the set of all elements of this sequence; in general, a sequence $\left(x_{n}\right)_{n \in \mathbb{N}}$ converges to $x$, if every monotone subsequence of this sequence converges to $x$. We say also that a sequence is convergent if it converges to some $x$. A linearly ordered set is said to be sequentially compact if each monotone sequence of its elements converges to some point in it. If a sequence $\left(x_{n}\right)_{n \in \mathbb{N}}$ converges to $x$, we denote this as usual by $\lim _{n \rightarrow \infty} x_{n}=x$. For a sequence $\left(x_{n}\right)_{n \in I}$ indexed by an infinite subset $I$ of $\mathbb{N}$, its convergence to a point $x$ is defined similarly and denoted by $\lim _{n \in I} x_{n}=x$. For an infinite $I \subseteq \mathbb{N}$ and a sequence $\left(f_{n}\right)_{n \in I}$ of functions from a set $X$ to a linearly ordered set $Y$, we say that $\left(f_{n}\right)_{n \in I}$ converges pointwise to $f: X \rightarrow Y$, if $\lim _{n \in I} f_{n}(x)=f(x)$ holds for every $x \in X$. We shall also say that a sequence $\left(f_{n}\right)_{n \in I}$ is pointwise convergent if there is some function $f$ to which the sequence converges pointwise.

Since any sequence in a linearly ordered set has a monotone subsequence, every sequence in a sequentially compact linearly ordered set has a convergent subsequence. Using this fact, we can see easily the following.

Lemma 1. If $\left(x_{n}\right)_{n \in \mathbb{N}}$ is a non-convergent sequence of elements of a sequentially compact linearly ordered set $X$, then there exist infinite subsets of natural numbers $I$ and $J$ such that subsequences $\left(x_{n}\right)_{n \in I}$ and $\left(x_{n}\right)_{n \in J}$ converge to different points of $X$.

Lemma 2. Any infinite linearly ordered set $X$ can be embedded into a dense linear order $\tilde{X}$ such that $d(\tilde{X})=d(X)$. If $X$ is sequentially compact, then $\tilde{X}$ can be also chosen to be so. Also, convergent sequences in $X$ remain convergent in $\tilde{X}$ with the same limit.

Proof. Let $D$ be a dense subset of $X$ of cardinality $d(X)$. For points $x, y \in X$, let us call $(x, y)$ a jump in $X$ if $x<y$ and there is no $z \in X$ such that $x<z<y$. By definition of dense subsets, for each jump $(x, y)$ one of the points $x$ or $y$ must be in $D$. Hence there are at most $d(X)$ jumps. Let $\tilde{X}$ be the linearly ordered set constructed from $X$ by inserting a copy of the reals into each of the jumps in $X$. Noting that the density of the reals $\mathbb{R}$, with respect to the canonical ordering, is countable and $\mathbb{R} \cup\{-\infty,+\infty\}$ is sequentially compact, it is easy to see that $\tilde{X}$ is as desired.

Lemma 3. Suppose that $X$ and $Y$ are linearly ordered sets and $\left(f_{n}\right)_{n \in I}$ is a sequence of increasing functions from $X$ to $Y$. If $\left(f_{n}\right)_{n \in I}$ converges pointwise to a function $f: X \rightarrow Y$, then $f$ is also increasing.

Proof. By Lemma 2, we may assume that $Y$ is a dense linear order. The rest of the proof can be done just like the usual proof of the corresponding assertion on increasing real functions.

Any linearly ordered set $X$ can be densely embedded into a sequentially compact linearly ordered set $\bar{X}$. E.g. we can take the Dedekind completion of $X$ as $\bar{X}$. Note that we have $d(X)=d(\bar{X})$ since $X$ is dense in $\bar{X}$. In general, the Dedekind completion of $X$ is not the minimal sequentially compact linearly ordered set containing a dense copy of $X$ since there can be an unfilled Dedekind cut $(D, E)$ of $X$ such that $D$ has uncountable cofinality and $E$ uncountable coinitiality. Let us call a sequence 
$\left(x_{n}\right)_{n \in \mathbb{N}}$ in a linearly ordered set $X$ potentially convergent if it converges to some point in some $\bar{X}$ as above. By virtue of Lemma 1, this is equivalent to saying that there are no $x, y \in X$ and no infinite $I, J \subseteq \mathbb{N}$ such that $x_{n} \leq x<y \leq x_{m}$ for every $n \in I$ and $m \in J$.

\section{The SPLitTing NUMBeR}

A family $\mathcal{S}$ of infinite subsets of $\mathbb{N}$ is said to be splitting if, for every infinite subset $I \subseteq \mathbb{N}$, there exists a set $J \in \mathcal{S}$ such that $I \cap J$ and $I \backslash J$ are both infinite. The splitting number $\mathbf{s}$ is defined by

$$
\mathbf{s}=\min \{|\mathcal{S}|: \mathcal{S} \text { is a splitting family }\} .
$$

In particular, if $\mathcal{S}$ is a family of infinite subsets of $\mathbb{N}$ of cardinality less than $\mathbf{s}$, then there exists an infinite subset $I \subseteq \mathbb{N}$ such that $I$ is almost included either in $J$ or in $\mathbb{N} \backslash J$ for every $J \in \mathcal{S}$. It is readily seen that $\mathbf{s}$ is uncountable and less than or equal to the cardinality of the reals. On the other hand, it is known that the value of $\mathbf{s}$ cannot be decided from the axioms of set theory alone. A splitting family was considered first by Sierpiński in [5]. He showed that under the Continuum Hypothesis there is a splitting family $\mathcal{S}$ with the property that every uncountable subfamily of $\mathcal{S}$ is still splitting. For more about the cardinal $\mathbf{s}$ and its relation to other cardinal invariants of reals the reader may consult [1], [2] or [7]. The role of splitting number in connection with convergence was also studied in [8].

The following lemma is the set-theoretic core of the generalization of Helly's theorem.

Lemma 4. If $X$ is a set of cardinality less than $\mathbf{s}$ and $Y$ is a sequentially compact linearly ordered set of density less than $\mathbf{s}$, then for any sequence $\left(f_{n}\right)_{n \in \mathbb{N}}$ of functions from $X$ to $Y$ there exists an infinite subset $I \subseteq \mathbb{N}$ such that the sequence of functions $\left(f_{n}\right)_{n \in I}$ converges pointwise.

Proof. By Lemma 2 we may assume that $Y$ is a dense linear order. Let $D$ be a dense subset of $Y$ of cardinality less than $\mathbf{s}$. For $x \in X$ and $y \in D$, let

$$
C_{x}^{y}=\left\{n \in \mathbb{N}: f_{n}(x)<y\right\} .
$$

Since $|X \times D|<\mathbf{s}$, there exists an infinite $I \subseteq \mathbb{N}$ such that $I$ is almost included either in $C_{x}^{y}$ or in $\mathbb{N} \backslash C_{x}^{y}$ for any $x \in X$ and $y \in D$.

We shall show that the set $I$ is as desired. Otherwise there would be some point $a \in X$ such that the sequence $\left(f_{n}(a)\right)_{n \in I}$ of points in $Y$ is not convergent. Then, by Lemma 1, there are infinite subsets $J$ and $K$ of $I$ and a point $d \in D$ such that sequences $\left(f_{n}(a)\right)_{n \in J}$ and $\left(f_{n}(a)\right)_{n \in K}$ of points in $Y$ are convergent and we have

$$
\lim _{n \in J} f_{n}(a)<d<\lim _{n \in K} f_{n}(a) .
$$

Hence we have $f_{n}(a)<d$ for all but finitely many $n \in J$ and $d<f_{n}(a)$ for all but finitely many $n \in K$. It follows that the sets $I \cap C_{a}^{d}$ and $I \backslash C_{a}^{d}$ are both infinite; but this contradicts the choice of $I$.

Lemma 4 gives the consistency of a positive answer to the following question of S. Saks studied in [5]:

For arbitrary sequence $\left(f_{n}\right)_{n \in \mathbb{N}}$ of real functions, do there exist an infinite $I \subseteq \mathbb{N}$ and an uncountable $X \subset \mathbb{R}$ such that, for each $x \in X$, the sequence of real numbers $\left(f_{n}(x)\right)_{n \in I}$ has a finite or infinite limit? 
Under the Continuum Hypothesis, Sierpiński gave a negative answer to the question in [5]. By applying Lemma 4 for the sequentially compact linearly ordered set $\mathbb{R} \cup\{-\infty,+\infty\}$, we see that, under $\mathbf{s}>\aleph_{1}$, a positive answer to the question is obtained.

Since every linearly ordered set can be embedded densely into a sequentially compact linearly ordered set, the next lemma follows immediately from Lemma 4 .

Lemma 5. If $X$ is a set of cardinality less than $\mathbf{s}$ and $Y$ is a linearly ordered set of density less than $\mathbf{s}$, then for any sequence $\left(f_{n}\right)_{n \in \mathbb{N}}$ of functions from $X$ to $Y$ there exists an infinite subset $I \subseteq \mathbb{N}$ such that the sequence $\left(f_{n}(x)\right)_{n \in I}$ is potentially convergent for every $x \in X$.

Lemma 5 can be yet slightly improved. For any infinite $I \subseteq \mathbb{N}$, let us call a sequence $\left(x_{n}\right)_{n \in I}$ in a linearly ordered set $X$ semi-monotone if there is a bijection $\varphi: \mathbb{N} \rightarrow I$ such that $\left(x_{\varphi(n)}\right)_{n \in \mathbb{N}}$ is eventually monotone, i.e. monotone from some $m \in \mathbb{N}$ on. It is clear that a semi-monotone sequence is potentially convergent. If $x=\lim _{n \in I} x_{n}$ exists, then $\left(x_{n}\right)_{n \in I}$ is semi-monotone if and only if $\left(x_{n}\right)_{n \in I}$ approaches to $x$ eventually from one side - i.e. for some $m \in \mathbb{N}$ either $x_{n} \leq x$ for every $n \geq m$ or $x \leq x_{n}$ for every $n \geq m$.

Lemma 6. If $X$ is a set of cardinality less than $\mathbf{s}$ and $Y$ is a linearly ordered set of density less than $\mathbf{s}$, then for any sequence $\left(f_{n}\right)_{n \in \mathbb{N}}$ of functions from $X$ to $Y$ there exists an infinite subset $I \subseteq \mathbb{N}$ such that the sequence $\left(f_{n}(x)\right)_{n \in I}$ is semi-monotone for every $x \in X$.

Proof. Without loss of generality, we may assume that $Y$ is sequentially compact. By Lemma 4 , there is an infinite $I \subseteq \mathbb{N}$ such that $\left(f_{n}\right)_{n \in I}$ is pointwise convergent. For each $x \in X$ let $y_{x}=\lim _{n \in I} f_{n}(x)$. Let $\tilde{Y}$ be the linearly ordered set obtained from $Y$ by inserting a new point $y_{x}^{\prime}$ between $y_{x}$ and $\left\{y \in Y: y_{x}<y\right\}$ for each $x \in X . \tilde{Y}$ is still sequentially compact and $d(\tilde{Y})<\mathbf{s}$ since only fewer than $\mathbf{s}$ new points are added. Hence we can apply Lemma 4 again to $\left(f_{n}\right)_{n \in I}$ as a sequence of functions from $X$ to $\tilde{Y}$ to obtain an infinite $J \subseteq I$ such that $\left(f_{n}\right)_{n \in J}$ is pointwise convergent as a sequence of functions from $X$ to $\tilde{Y}$. For each $x \in X$, as $\left(f_{n}(x)\right)_{n \in J}$ should converge to $y_{x}$ or $y_{x}^{\prime}$, it follows that, for each $x \in X,\left(f_{n}(x)\right)_{n \in J}$ as a sequence of points in $Y$ approaches $y_{x}$ eventually from one side. Hence by the remark before this lemma, $\left(f_{n}(x)\right)_{n \in J}$ is a quasi-monotone sequence in $Y$.

\section{Generalized Helly's theorem}

Since the density of the reals is countable, Helly's theorem ([3]) as cited in the Introduction is just a special case of the following theorem.

Theorem 7 (Generalized Helly's Theorem). Let $X$ and $Y$ be linearly ordered sets. If $Y$ is sequentially compact with density less than $\mathbf{s}$, then any sequence of monotone functions from $X$ to $Y$ contains a pointwise convergent subsequence.

Proof. Without loss of generality, we may assume that the sequence $\left(f_{n}\right)_{n \in \mathbb{N}}$ consists of increasing functions. By Lemma 2 we may also assume that $Y$ is a dense linear order. Let $D \subseteq Y$ be a dense subset of cardinality less than s. For $b, d \in D$ and $n \in \mathbb{N}$, let $x_{b, d}^{n}$ be an element of $X$ such that $b \leq f_{n}\left(x_{b, d}^{n}\right) \leq d$ if such an element exists; otherwise let $x_{b, d}^{n}$ be an arbitrary element of $X$. Let

$$
Z=\left\{x_{b, d}^{n}: b, d \in D, n \in \mathbb{N}\right\} .
$$


Then we have $|Z|<\mathbf{s}$. Hence, by Lemma 4 , there exists an infinite subset $L \subseteq \mathbb{N}$ such that the sequence of functions $\left(f_{n}\lceil Z)_{n \in L}\right.$ converges pointwise where $f_{n}\lceil Z$ denotes the restriction of the function $f_{n}$ to the set $Z$. Let

$$
T=\left\{x \in X:\left(f_{n}(x)\right)_{n \in L} \text { is a convergent sequence of points in } Y\right\} .
$$

We have $Z \subseteq T$. Let $h: T \rightarrow Y$ be the function such that the sequence of functions $\left(f_{n}\lceil T)_{n \in L}\right.$ converges pointwise to $h$. By Lemma $3, h$ is an increasing function. Now let

$$
\mathcal{U}=\{U: U \text { is a maximal interval in } X \text { such that } U \subseteq X \backslash T\} .
$$

For each interval $U \in \mathcal{U}$ we choose $x_{U} \in U$. By definition of $Z, f_{n}$ is constant on each $U \in \mathcal{U}$ for every $n \in \mathbb{N}$. Hence, for any subset $M \subseteq L$, we have:

$(*) \quad\left(f_{n}\left(x_{U}\right)\right)_{n \in M}$ converges if and only if $\left(f_{n}\lceil U)_{n \in M}\right.$ converges pointwise.

Letting $W=\left\{x_{U}: U \in \mathcal{U}\right\}$, we claim that $|W|<\mathbf{s}$. To see this, let $x \in$ $W$. The sequence $\left(f_{n}(x)\right)_{n \in L}$ is not convergent. Since $Y$ is a dense linear order and sequentially compact, by Lemma 1 , there are infinite subsets $J, K \subseteq L$ and points $b_{x}, c_{x}, d_{x} \in D$ such that sequences of points $\left(f_{n}(x)\right)_{n \in J}$ and $\left(f_{n}(x)\right)_{n \in K}$ are convergent and

$$
\lim _{n \in J} f_{n}(x)<b_{x}<d_{x}<c_{x}<\lim _{n \in K} f_{n}(x) .
$$

If $y \in T$ and $y<x$, then $h(y) \leq b_{x}$, since $\lim _{n \in L} f_{n}(y)=h(y)$ and $f_{n}(y)<b_{x}$ for infinitely many $n \in L$. Likewise, for any $z \in T$ with $x<z$ we have $h(z) \geq c_{x}$. For $x_{1}, x_{2} \in W$ with $x_{1}<x_{2}$, there is $y \in T$ such that $x_{1}<y<x_{2}$. Hence the mapping from $W$ to $D$ defined by $x \mapsto d_{x}$ is injective. As $|D|<\mathbf{s}$, it follows that $|W|<\mathbf{s}$.

Again by Lemma 4 we can find an infinite $I \subseteq L$ such that $\left(f_{n} \uparrow W\right)_{n \in I}$ is pointwise convergent. By definitions and $(*)$ above, we have that the sequence of functions $\left(f_{n}\right)_{n \in I}$ converges pointwise.

\section{The SPlitting NUMBer is OPtimal}

For an infinite subset $V \subseteq \mathbb{N}$, let

$$
\mathcal{D}(V)=\sum_{n \in V} \frac{1}{2^{n+1}}
$$

Note that $\mathcal{D}$ is a bijective mapping from infinite subsets of $\mathbb{N}$ to the real numbers in the half-open interval $(0,1]$. For a family $\mathcal{S}$ of subsets of $\mathbb{N}$, let us denote by $\mathcal{D}(\mathcal{S})$ the set $\{\mathcal{D}(V): V \in \mathcal{S}\}$. Thus $\mathcal{D}(\mathcal{S})$ is a subset of the unit interval of cardinality $|\mathcal{S}|$.

Assume now that $\mathcal{S}$ is a splitting family of cardinality $\mathbf{s}$. Let

$$
H=(\mathcal{D}(\mathcal{S}) \times[0,1]) \cup([0,1] \times\{0\})
$$

be the linearly ordered set equipped with the lexicographical ordering, i.e. we let $(x, y)<(p, q)$, whenever $x<p$, or $x=p$ and $y<q$. Here, $x<p$ and $y<q$ denote the canonical ordering on the reals.

Lemma 8. The linearly ordered set $H$ is sequentially compact and its density is equal to $\mathbf{s}$. 
Proof. Suppose that $S=\left(\left(x_{n}, y_{n}\right)\right)_{n \in \mathbb{N}}$ is a monotone sequence of points in $H$. Then $\left(x_{n}\right)_{n \in \mathbb{N}}$ is monotone as well. If $\left(x_{n}\right)_{n \in \mathbb{N}}$ is eventually constant, say $x_{n}=x$ for all $n>m$, then $\left(y_{n}\right)_{n>m}$ is a monotone sequence. Hence $\lim _{n \rightarrow \infty} y_{n}$ exists and $S$ converges to $\left(x, \lim _{n \rightarrow \infty} y_{n}\right)$. Otherwise there are infinitely many distinct $x_{n}$ 's. If $S$ is increasing, then $S$ converges to $\left(\lim _{n \rightarrow \infty} x_{n}, 0\right)$. If $S$ is decreasing, then $S$ converges to $\left(\lim _{n \rightarrow \infty} x_{n}, 1\right)$ provided that $\lim _{n \rightarrow \infty} x_{n} \in \mathcal{D}(\mathcal{S})$; otherwise it converges to $\left(\lim _{n \rightarrow \infty} x_{n}, 0\right)$.

Let $Q$ be the set of rational numbers in the unit interval $[0,1]$. Then

$$
H_{0}=(\mathcal{D}(\mathcal{S}) \times Q) \cup(Q \times\{0\})
$$

is dense in $H$ and of cardinality $\mathbf{s}$. This shows that $d(H) \leq \mathbf{s}$. If $H^{\prime} \subseteq H$ is of cardinality less than $\mathbf{s}$, then there is some $s \in \mathcal{D}(\mathcal{S})$ such that $\{s\} \times[0,1]$ is disjoint from $H^{\prime}$. Hence $H^{\prime}$ is not dense in $H$. Thus we also have $d(H) \geq \mathbf{s}$.

The following theorem is a variation of an example in [6].

Theorem 9. There exists a sequence $\left(f_{n}\right)_{n \in \mathbb{N}}$ of increasing functions from the subset $\mathcal{D}(\mathcal{S})$ of the unit interval to the linearly ordered set $H$ such that $\left(f_{n}\right)_{n \in \mathbb{N}}$ does not have any pointwise convergent subsequence.

Proof. For each $n \in \mathbb{N}$ and $V \in \mathcal{S}$, let

$$
f_{n}(\mathcal{D}(V))= \begin{cases}(\mathcal{D}(V), 1), & \text { if } n \in V ; \\ (\mathcal{D}(V), 0), & \text { otherwise. }\end{cases}
$$

Each function $f_{n}$ is obviously increasing. For any infinite subsequence $\left(f_{n}\right)_{n \in I}$, let $V \in \mathcal{S}$ be such that the sets $I \cap V$ and $I \backslash V$ are both infinite. Then we have $f_{n}(\mathcal{D}(V))=(\mathcal{D}(V), 1)$ for every $n \in I \cap V$, and $f_{n}(\mathcal{D}(V))=(\mathcal{D}(V), 0)$ for every $n \in I \backslash V$. In particular, the sequence of points $\left(f_{n}(\mathcal{D}(V))\right)_{n \in I}$ is not convergent.

The theorem above shows that the condition $d(Y)<\mathbf{s}$ in Theorem 7 is optimal. Using this fact, we obtain the following characterization of the splitting number.

Let $\tau_{1}$ be the supremum of the cardinals $\kappa$ with the property that for every set of $X$ of cardinality less than $\kappa$ and for every sequentially compact linearly ordered set $Y$ of density less than $\kappa$, any sequence of functions from $X$ to $Y$ has a pointwise convergent subsequence. Likewise, let $\tau_{2}$ be the least cardinal $\kappa$ such that, for some set $X$ of cardinality $\kappa$, it is not the case that any sequence of functions from $X$ to $\{0,1\}$ has a pointwise convergent subsequence, where we consider $\{0,1\}$ as a linearly ordered set with $0<1$. Finally, let $\mu$ be the supremum of the cardinals $\kappa$ with the property that, for any linearly ordered set $X$ and any sequentially compact linearly ordered set $Y$ with $d(Y)<\kappa$, any sequence of monotone functions from $X$ to $Y$ has a pointwise convergent subsequence.

Theorem 10. $\mathbf{s}=\tau_{1}=\tau_{2}=\mu$.

Proof. By definition we have $\tau_{1} \leq \tau_{2}$. Lemma 4 implies $\mathbf{s} \leq \tau_{1} ; \mathbf{s} \leq \mu$ follows from Theorem 7. Theorem 9 implies $\mathbf{s} \geq \mu$.

To see $\tau_{2} \leq \mathbf{s}$, we use a variant of Rademacher's functions (see [4]): for each $n \in \mathbb{N}$, the function $\varphi_{n}$ on the family of all infinite subsets of $\mathbb{N}$ to $\{0,1\}$ is defined by

$$
\varphi_{n}(V)= \begin{cases}1, & \text { if } n \in V \\ 0, & \text { otherwise }\end{cases}
$$


Let $\mathcal{S}$ be a splitting family of cardinality s. For any infinite subset $I \subseteq \mathbb{N}$, let $V \in \mathcal{S}$ be such that the sets $I \cap V$ and $I \backslash V$ are both infinite. Then the $0-1$ sequence $\left(\varphi_{n}(V)\right)_{n \in I}$ is not convergent as 0 and 1 both appear infinitely many times in this sequence. This shows that no subsequence of the sequence $\left(\varphi_{n}\lceil\mathcal{S})_{n \in \mathbb{N}}\right.$ of functions from $\mathcal{S}$ to $\{0,1\}$ can be pointwise convergent.

\section{REFERENCES}

[1] B. Balcar, P. Simon, Disjoint refinement, in: Handbook of Boolean Algebras, edited by J. D. Monk with R. Bonnet, Elsevier Science Publishers B.V. (1989), 333-386.

[2] E.K. van Douwen, The integers and topology, in: Handbook of Set-Theoretic Topology, edited by K. Kunen and J.E. Vaughan, North-Holland (1984), 111-167. MR 87f:54008

[3] E. Helly, Über lineare Funktionaloperationen, Sitzungsberichte der Naturwiss. Klasse Kais. Akad. Wiss., Wien 121 (1921), 265-295.

[4] H. Rademacher, Einige Sätze über Reihen von allgemeinen Orthogonalfunktionen, Math. Ann. 87 (1922), 112-138.

[5] W. Sierpiński, Remarque sur les suites infinies de fonctions, Fund. Math. XVIII (1932), 110113.

[6] A. Tychonoff, Über einen Funktionenraum, Math. Ann. 111 (1935), 762-766.

[7] J.E. Vaughan, Small uncountable cardinals and topology, in: Open Problems in Topology, edited by J. van Mill and G.M. Reed, North-Holland (1990), 195-218. CMP 91:03

[8] P. Vojtás, More on set-theoretic characteristics of summability of sequences by regular (Toeplitz) matrices, Comm. Math. Univ. Carolinae 29 (1988), 97-102. MR 89e:03090

Institut für Mathematik II, Freie Universität Berlin, Arnimallee 3, 14195 Berlin, Germany

E-mail address: fuchino@math.fu-berlin.de

Current address: Department of Computer Sciences, Kitami Institute of Technology, Kitami, Hokkaido 090 Japan

E-mail address: fuchino@math.cs.kitami-it.ac.jp

Instytut Matematyki Uniwersytetu Ślasskiego, ul. Bankowa 14, 40007 Katowice, POLAND

E-mail address: plewik@ux2.math.us.edu.pl 